\title{
Perioperative Laboratory Abnormalities in Gynecologic Oncology Surgical Patients
}

\author{
Tatnai L. Burnett, MD, ${ }^{1 *}$ Justin Junn, MD, ${ }^{1 \dagger}$ Giselle E. Kolenic, MA, ${ }^{2}$ Catherine Christen, PharmD, ${ }^{3}$ \\ Carolyn M. Johnston, MD, R. Kevin Reynolds, MD, and Karen McLean, MD, PhD ${ }^{1}$
}

\begin{abstract}
Background: Laboratory blood testing incurs financial costs and the blood draws can increase discomfort, yet minimal data exists regarding routine testing in gynecologic oncology surgical patients. Additionally, an increasing number of gynecologic oncology surgeries are performed via a laparoscopic approach. Thus, further investigation into perioperative laboratory testing for gynecologic oncology patients is warranted. An increasing number of gynecologic oncology surgeries are performed via a laparoscopic approach. Thus, further investigation into perioperative laboratory testing for gynecologic oncology patients is warranted. Objective: The aims of this study were (1) to evaluate the frequency and etiology of perioperative laboratory test abnormalities in patients undergoing laparoscopic and laparotomy surgery in a gynecologic oncology service, and (2) to establish an evidence-based algorithm to reduce unnecessary laboratory testing. Materials and Methods: A single-institution retrospective study was completed, investigating laparoscopic and laparotomic surgeries over 4 years. Information on preoperative and postoperative laboratory data, surgical parameters, perioperative interventions, and patient demographics was collected. Quality-assurance data were reviewed. Data were tabulated and analyzed using Statistical Product and Service Solutions (SPSS) version 22. A Student's $t$-test was used to test for group differences for continuous variables with equal variance, the Mann-Whitney- $U$ test for continuous variables when unequal variance was detected, and Pearson's $\chi^{2}$ was used to investigate categorical variables of interest. $p$-Values $<0.05$ were considered to be statistically significant. Logistic regression was performed to investigate the relationships among multiple predictors and each identified outcome. Results: The study included 481 subjects (168 laparoscopies, 313 laparotomies). Patients undergoing laparoscopy were, on average, younger (53.5 versus 57.4 ), with lower body mass indexes (29.7 versus 33.0 ) and lower rates of diabetes (10.7\% versus $19.5 \%$ ), compared to patients undergoing laparotomy. Overall, $>98 \%$ of patients underwent at least one preoperative and postoperative laboratory test, totaling 8060 preoperative and 5784 postoperative results. The laparoscopy group was significantly less likely to have postoperative metabolic abnormalities or to undergo perioperative blood transfusion. Patients taking an angiotensin-converting-enzyme inhibitor, angiotensin-II-receptor blocker, or diuretic were significantly more likely to have elevated creatinine preoperatively (odds ratio [OR]: $5.0 ; p<0.001$ ) and postoperatively (OR: $7.1 ; p<0.001$ ), and this remained true for each group when divided by surgical approach. Perioperative complications meeting institutional quality assurance criteria occurred in $1.7 \%$ of laparoscopy patients compared to $11.8 \%$ of laparotomy patients $(p<0.001)$; perioperative laboratory testing was not a factor in the diagnosis of these complications. Conclusions: Clinically significant laboratory abnormalities are uncommon and are less likely to be found on routine perioperative testing in gynecologic oncology patients undergoing laparoscopy, compared to patients undergoing laparotomy. This suggests a role for limiting perioperative laboratory blood testing. ( J GYNECOL SURG 32:111)
\end{abstract}

\footnotetext{
${ }^{1}$ Division of Gynecologic Oncology, Department of Obstetrics and Gynecology, ${ }^{2}$ Center for Statistical Consultation and Research, and ${ }^{3}$ College of Pharmacy, University of Michigan, Ann Arbor, MI.

*Current affiliation: Department of Obstetrics and Gynecology, Mayo Clinic, Rochester, MN.

$\nmid$ Current affiliation: Department of Obstetrics and Gynecology, University of Washington, Seattle, WA.

(C) Tatnai L. Burnett et al., 2016; Published by Mary Ann Liebert, Inc. This Open Access article is distributed under the terms of the Creative Commons Attribution Noncommercial License (http://creativecommons.org/licenses/by-nc/4.0/) which permits any noncommercial use, distribution, and reproduction in any medium, provided the original author(s) and the source are credited.
} 


\section{Introduction}

$\mathbf{T}$ HE RATIONALE AND UTILITY of perioperative blood testing in the gynecologic oncology patient population has received minimal evaluation in the literature. Laboratory blood testing is associated with financial costs and requires patients to undergo blood draws that can increase discomfort. Gynecologic oncology is also experiencing a significant shift in surgical approach, with an increasing number of surgeries completed by laparoscopic approach and an associated decrease in conventional open cases. ${ }^{1}$ Thus, further investigation into perioperative laboratory testing in the gynecologic oncology patient population is warranted.

The objectives of this study were (1) to assess the frequency and clinical significance of perioperative laboratory testing abnormalities in gynecologic oncology patients undergoing laparoscopic and laparotomic surgery, with the goal of identifying and eliminating unnecessary testing, and (2) to establish an evidence-based algorithm to reduce unnecessary laboratory testing. It was hypothesized that (1) more laboratory tests are obtained than are necessary for clinical management, (2) the majority of abnormal laboratory results are not clinically significant and do not result in a change in management, and (3) laboratory result abnormalities would be less frequent among patients undergoing laparoscopy. Using these findings, the current authors propose an algorithm for streamlining perioperative testing in gynecologic oncology surgical patients.

\section{Materials and Methods}

A retrospective study was performed. Institutional Review Board Project \#HUM00045862 was approved on April 19, 2011. Surgical cases performed by three gynecologic oncology attending physicians at the University of Michigan Medical Center, in Ann Arbor, from March 6, 2006, to March 6, 2010 were identified. Patients were included if they underwent laparoscopy or laparotomy. Patients were excluded if they were admitted prior to the date of their operation, if bowel obstruction was the indication for surgery, if patients were on total parenteral nutrition prior to surgery, or if they were pregnant or within 6 weeks postpartum. For patients with more than one surgery, only the initial procedure was included. Cases converted from laparoscopy to laparotomy were categorized as laparotomy.

Patient characteristics were documented, including age, body mass index (BMI), race, and final pathologic diagnosis. Information on the presence of cardiovascular disease and/or diabetes mellitus was collected from patient preoperative history and physical documentation; home medications recorded included angiotensin-converting-enzyme (ACE) inhibitors, angiotensin-II-receptor blockers (ARB), insulin, oral hypoglycemics, diuretics, and potassium supplements. Preoperative mechanical bowel preparation was also recorded. All records were searched for preoperative and postoperative day 1 blood laboratory testing results, and these values were recorded. Hospital and postoperative visit records were searched for information on postoperative infections and antibiotic use.

Departmental quality-assurance records were reviewed to determine the number of cases that met quality-assurance criteria. Each case was then reviewed to determine if and how laboratory results factored into the identification and management of the case. Data were tabulated and analyzed using Statistical Product and Service Solutions (SPSS), version 22. Student's $t$-test was used to test for group differences for continuous variables with equal variance, Mann-Whitney- $U$ test for continuous variables when unequal variance was detected, and Pearson's $\chi^{2}$ was used to investigate categorical variables of interest. $p$-Values $<0.05$ were considered to be statistically significant. Logistic regression was performed to investigate the relationships among multiple predictors and each identified outcome.

\section{Results}

\section{Characteristics of the patient cohort}

The study involved 481 patients who met the inclusion criteria, with 168 laparoscopy cases and 313 laparotomy cases. Patient characteristics are listed in Table 1. There was no statistically significant difference in surgical approach (laparoscopy versus laparotomy) for any race. The average BMI for all patients was $31.8 \mathrm{~kg} / \mathrm{m}^{2}$; patients with a $\mathrm{BMI}>40 \mathrm{~kg} / \mathrm{m}^{2}$ had 5 times the odds of undergoing surgery via laparotomy, compared to laparoscopy $(p=0.047)$. Final pathologic diagnosis was malignancy in $85.1 \%$ of laparoscopies and in $74.1 \%$ of laparotomies, for a total of $78 \%$ of the cases.

Cardiovascular disease was reported in $7.3 \%$ of the overall patient population, and no statistical difference was noted between the laparoscopy and laparotomy cohorts (Table 1). Approximately $37 \%$ of patients reported taking either an ACE inhibitor/ARB or a diuretic, which were termed, collectively, as "renally active medications." A statistically significant difference according to surgical approach was observed, with a higher percentage of these patients undergoing laparotomy $(p=0.008$ for ACE inhibitor/ARB; $p=0.018$ for diuretic; Table 1$)$.

Diabetes mellitus was reported in $16.4 \%$ of the patients, including $10.7 \%$ of patients undergoing laparoscopy and $19.5 \%$ of patients undergoing laparotomy $(p=0.014$; Table 1). Only a subset of diabetic patients was taking insulin (3.3\% of all patients), and these patients underwent laparotomy more often than laparoscopy $(p=0.014$; Table 1). A larger percentage of patients $(13.1 \%)$ were taking oral hypoglycemic agents, with no statistically significant difference in surgical approach.

Surgical parameters were reviewed and compared among the cohorts (Table 2). Cases performed via laparoscopy were longer in total operative time ( $\sim 4$ hours versus 3 hours for laparotomy; $p<0.001)$. Estimated blood loss (EBL) was lower for laparoscopy, and blood transfusion occurred in a total of 59 patients $(12.3 \%)$, only 1 of whom underwent laparoscopy $(p<0.001)$.

\section{Frequency of laboratory testing and incidence of abnormalities}

The number of patients undergoing laboratory testing is detailed in Table 3, including preoperative and postoperative comprehensive or basic metabolic panels, magnesium, phosphate, and complete blood count with platelets. Overall, $>98 \%$ of patients underwent at least one preoperative and postoperative laboratory test, totaling 8060 preoperative and 5784 postoperative individual laboratory results. Of these results, $11.8 \%$ of all preoperative laboratory values were 
Table 1. Patients' Characteristics According to Surgical Approach

\begin{tabular}{|c|c|c|c|c|}
\hline Characteristics & All patients $(\mathrm{N}=481)$ & Laparoscopy $(\mathrm{n}=168)$ & Laparotomy $(\mathrm{n}=313)$ & $\mathrm{p}-$ Value $^{\mathrm{a}}$ \\
\hline Age & 56.05 (SD: 14.07) & 53.46 (SD: 14.63) & 57.44 (SD: 13.58) & 0.003 \\
\hline BMI & 31.83 (SD: 9.69) & 29.74 (SD: 6.93) & 32.96 (SD: 10.73) & $<0.001$ \\
\hline \multicolumn{5}{|l|}{ Race } \\
\hline White or Caucasian & $439(91.3 \%)$ & $154(91.7 \%)$ & $285(91.1 \%)$ & 0.867 \\
\hline Black or African American & $18(3.7 \%)$ & $5(3.0 \%)$ & $13(4.0 \%)$ & 0.622 \\
\hline Asian & $10(2.1 \%)$ & $4(2.4 \%)$ & $6(1.9 \%)$ & 0.743 \\
\hline $\begin{array}{l}\text { Native Hawaiian \& other } \\
\text { Pacific Islander }\end{array}$ & $2(0.4 \%)$ & $0(0.0 \%)$ & $2(0.6 \%)$ & 0.547 \\
\hline Hispanic & $1(0.2 \%)$ & $1(0.6 \%)$ & $0(0.0 \%)$ & 0.345 \\
\hline Other & $4(0.8 \%)$ & $1(0.6 \%)$ & $3(1.0 \%)$ & 1.000 \\
\hline Unknown & $7(1.5 \%)$ & $3(1.8 \%)$ & $4(1.3 \%)$ & 1.000 \\
\hline \multicolumn{5}{|l|}{ Diagnosis $^{\mathrm{b}}$} \\
\hline Endometrial cancer & $189(39.3 \%)$ & $88(52.4 \%)$ & $101(32.3 \%)$ & $<0.001$ \\
\hline Ovarian cancer & $111(23.1 \%)$ & $14(8.3 \%)$ & $97(31.0 \%)$ & $<0.001$ \\
\hline Cervical cancer & $56(11.6 \%)$ & $37(22.0 \%)$ & $19(6.1 \%)$ & $<0.001$ \\
\hline Other malignancy & $25(5.2 \%)$ & $6(3.6 \%)$ & $19(6.1 \%)$ & 0.286 \\
\hline Benign & $106(22.0 \%)$ & $25(14.9 \%)$ & $81(25.9 \%)$ & 0.006 \\
\hline \multicolumn{5}{|l|}{ Comorbidities } \\
\hline Cardiovascular disease & $35(7.3 \%)$ & $9(5.4 \%)$ & $26(8.3 \%)$ & 0.273 \\
\hline Diabetes mellitus & $79(16.4 \%)$ & $18(10.7 \%)$ & $61(19.5 \%)$ & 0.014 \\
\hline \multicolumn{5}{|l|}{ Medications } \\
\hline ACEi/ARB & $120(24.9 \%)$ & $30(17.9 \%)$ & $90(28.8 \%)$ & 0.008 \\
\hline Diuretic & $113(23.5 \%)$ & $29(17.3 \%)$ & $84(26.8 \%)$ & 0.018 \\
\hline Potassium & $17(3.5 \%)$ & $3(1.8 \%)$ & $14(4.5 \%)$ & 0.194 \\
\hline Insulin & $16(3.3 \%)$ & $1(0.6 \%)$ & $15(4.8 \%)$ & 0.014 \\
\hline Oral hypoglycemic & $63(13.1 \%)$ & $17(10.1 \%)$ & $46(14.7 \%)$ & 0.202 \\
\hline
\end{tabular}

Age and BMI values are listed as mean (SD), all others are $n$ (percentage).

${ }^{\text {a }}$ Statistically significant $p$-values are indicated in bold.

${ }^{\mathrm{b}}$ Percentages sum to $>100 \%$, as 6 patients had diagnoses of synchronous endometrial and ovarian cancers.

BMI, body mass index; SD, standard deviation; ACEi, angiotensin-converting-enzyme inhibitor; ARB, angiotensin-II-receptor blocker.

outside the normal reporting range and $36.6 \%$ of postoperative laboratory results were returned outside the normal range. The mean number of abnormal laboratory results per patient was 2.0 (standard deviation [SD]: 2.0) preoperatively and 4.5 (SD: 2.1 ) on postoperative day 1 . When divided by cohort, the laparotomy group had a significantly higher mean number of abnormal laboratory results per patient; preoperatively 2.4 (SD: 2.1) for laparotomy versus 1.3 (SD 1.4) for laparoscopy, and postoperatively 4.9 (SD: 2.1$)$ for laparotomy, compared to 3.7 (SD: 1.9) for laparoscopy $(p<$ 0.001 for both comparisons).

Preoperatively, 412 of the 481 women underwent a comprehensive metabolic panel $(85.7 \%)$ and an additional
45 women underwent a basic metabolic panel $(9.4 \%)$, for a total of $95.0 \%$ of women undergoing some type of preoperative metabolic testing. A complete blood count with platelets was tested in all but 8 women (98.3\%).

Laboratory test results were considered to be abnormal if they fell outside of the University of Michigan laboratory reference range (Table 3). Despite the high frequency of preoperative testing, the overall incidence of laboratory value abnormalities did not exceed $5 \%$ for the majority of the tests. Potentially clinically significant findings are indicated in bold in Table 3. Specifically, 44 patients $(9.6 \%)$ had elevated creatinine and 120 patients $(26.2 \%)$ had elevated glucose.

Table 2. Surgical Data and Blood Transfusions According to Surgical Approach

\begin{tabular}{lcccc}
\hline Factors & $\mathrm{n}^{\mathrm{a}}$ & All patients & Laparoscopy & Laparotomy \\
\hline Surgical time & 481 & 199.9 minutes (SD: 77.7) & 238.1 minutes (SD: 71.8) & 179.4 minutes (SD: 72.9) \\
EBL & 481 & 239.5 mL (SD: 443.1) & 97.2 mL (SD: 158.8) & $315.9 \mathrm{~mL}$ (SD: 521.3) \\
Perioperative transfusion & 481 & $59(12.3 \%)$ & $1(0.6 \%)$ & $58(18.5 \%)$ \\
$\quad$ Intraoperative $_{\text {Postoperative }}^{4}$ & 471 & $29(6.0 \%)$ & $1(0.6 \%)$ & $28(8.9 \%)$ \\
& 47 & $42(8.9 \%)$ & $1(0.6 \%)$ & $41(13.4 \%)$ \\
\hline
\end{tabular}

Data are mean (SD), or $n(\%)$, or $n$.

All comparisons of laparotomy to laparoscopy were statistically significant with $\mathrm{p}<0.001$.

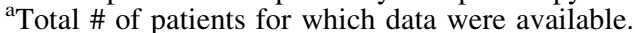

${ }^{\mathrm{b}}$ Some patients underwent both intraoperative and postoperative blood transfusions.

$\mathrm{SD}$, standard deviation; EBL, estimated blood loss. 
Table 3. Perioperative Laboratory Results for All Patients

\begin{tabular}{|c|c|c|c|c|c|c|c|}
\hline \multirow[b]{2}{*}{ Test } & \multirow[b]{2}{*}{ Normal range ${ }^{\mathrm{a}}$} & \multicolumn{3}{|c|}{ Preoperative testing } & \multicolumn{3}{|c|}{ Postoperative day 1 testing } \\
\hline & & $n^{\mathrm{b}}(\%)$ & $\begin{array}{c}\text { High } \\
\text { values (\%) }\end{array}$ & $\begin{array}{c}\text { Low } \\
\text { values (\%) }\end{array}$ & $n^{\mathrm{b}}(\%)$ & $\begin{array}{c}\text { High } \\
\text { values (\%) }\end{array}$ & $\begin{array}{c}\text { Low } \\
\text { values }(\%)\end{array}$ \\
\hline Sodium & $136-146 \mathrm{mmol} / \mathrm{L}$ & $457(95.0 \%)$ & 0 & $22(4.6 \%)$ & $383(79.6 \%)$ & $3(0.8 \%)$ & $32(8.4 \%)$ \\
\hline Potassium & $3.5-5.0 \mathrm{mmol} / \mathrm{L}$ & $457(95.0 \%)$ & $18(3.9 \%)$ & $11(2.4 \%)$ & $383(79.6 \%)$ & $17(4.4 \%)$ & $6(1.6 \%)$ \\
\hline Chloride & $98-108 \mathrm{mmol} / \mathrm{L}$ & $457(95.0 \%)$ & $28(6.1 \%)$ & $12(2.6 \%)$ & $383(79.6 \%)$ & $89(23.2 \%)$ & $4(1.0 \%)$ \\
\hline $\mathrm{CO}_{2}$ & $22-34 \mathrm{mmol} / \mathrm{L}$ & $457(95.0 \%)$ & $5(1.1 \%)$ & $9(2.0 \%)$ & $383(79.6 \%)$ & $2(0.5 \%)$ & $11(2.9 \%)$ \\
\hline UN & $8-20 \mathrm{mg} / \mathrm{dL}$ & $457(95.0 \%)$ & $67(14.7 \%)$ & $11(2.4 \%)$ & $383(79.6 \%)$ & $23(6.0 \%)$ & $101(26.4 \%)$ \\
\hline Creatinine & $0.5-1.0 \mathrm{mg} / \mathrm{dL}$ & $458(95.2 \%)$ & $44(9.6 \%)$ & $4(0.9 \%)$ & $383(79.6 \%)$ & $26(6.8 \%)$ & $22(5.7 \%)$ \\
\hline Glucose & $73-110 \mathrm{mg} / \mathrm{dL}$ & $458(95.2 \%)$ & $120(26.2 \%)$ & $9(2.0 \%)$ & $383(79.6 \%)$ & $318(83.0 \%)$ & $1(0.3 \%)$ \\
\hline Calcium & $8.6-10.3 \mathrm{mg} / \mathrm{dL}$ & $457(95.0 \%)$ & $15(3.3 \%)$ & $8(1.8 \%)$ & $383(79.6 \%)$ & $1(0.3 \%)$ & $264(68.9 \%)$ \\
\hline Protein & $6.0-8.3 \mathrm{~g} / \mathrm{dL}$ & $412(85.7 \%)$ & $1(0.2 \%)$ & $9(2.2 \%)$ & $27(5.5 \%)$ & 0 & $20(74.1 \%)$ \\
\hline Albumin & $3.5-4.9 \mathrm{~g} / \mathrm{dL}$ & $414(86.1 \%)$ & $5(1.2 \%)$ & $10(2.4 \%)$ & $28(5.7 \%)$ & 0 & $18(64.3 \%)$ \\
\hline AST & $8-30 \mathrm{IU} / \mathrm{L}$ & $412(85.7 \%)$ & $62(15.0 \%)$ & $1(0.2 \%)$ & $27(5.5 \%)$ & $8(29.6 \%)$ & $2(7.4 \%)$ \\
\hline ALT & $7-35 \mathrm{IU} / \mathrm{L}$ & $412(85.7 \%)$ & $53(12.9 \%)$ & $7(1.7 \%)$ & $27(5.5 \%)$ & $5(18.5 \%)$ & $2(7.4 \%)$ \\
\hline Alk & $30-130 \mathrm{IU} / \mathrm{L}$ & $412(85.7 \%)$ & $28(6.8 \%)$ & $1(0.2 \%)$ & $27(5.5 \%)$ & $2(7.4 \%)$ & $2(7.4 \%)$ \\
\hline Total bilirubin & $0.2-1.2 \mathrm{mg} / \mathrm{dL}$ & $412(85.7 \%)$ & $6(1.5 \%)$ & $2(0.5 \%)$ & $27(5.5$ & $2(7$. & 0 \\
\hline Magnesium & $2.7-4.6 \mathrm{mg} / \mathrm{dL}$ & $19(4.0 \%)$ & $1(5.3 \%)$ & 0 & $342(69.8 \%)$ & $8(2.4 \%)$ & $12(3.6 \%)$ \\
\hline Phosphate & $1.5-2.4 \mathrm{mg} / \mathrm{dL}$ & $18(3.7 \%)$ & $1(5.6 \%)$ & $2(11.1 \%)$ & $340(69.4 \%)$ & $13(3.9 \%)$ & $71(21.3 \%)$ \\
\hline $\begin{array}{l}\text { White blood } \\
\text { cells }\end{array}$ & $4.0-10.0 \mathrm{~K} / \mathrm{mm}^{3}$ & $473(98.3 \%)$ & $50(10.6 \%)$ & $17(3.6 \%)$ & $472(98.1 \%)$ & $223(47.2 \%)$ & $2(0.4 \%)$ \\
\hline Hemoglobin & $12.0-16.0 \mathrm{~g} / \mathrm{dL}$ & $473(98.3 \%)$ & $3(0.6 \%)$ & $107(22.6 \%)$ & $472(98.1 \%)$ & $1(0.2 \%)$ & $355(75.2 \%)$ \\
\hline Hematocrit & $36.0 \%-48.0 \%$ & $473(98.3 \%)$ & 0 & $133(28.1 \%)$ & $472(98.1 \%)$ & $1(0.2 \%)$ & $394(83.5 \%)$ \\
\hline Platelets & $150-400 \mathrm{~K} / \mathrm{mm}^{3}$ & $472(98.1 \%)$ & $54(11.4 \%)$ & $16(3.4 \%)$ & $471(97.9 \%)$ & $21(4.5 \%)$ & $34(7.2 \%)$ \\
\hline
\end{tabular}

Data are $\mathrm{n}(\%)$, or $\mathrm{n}$

Potentially clinically significant findings are indicated in bold.

${ }^{a}$ Normal range per University of Michigan, Ann Arbor, laboratory.

${ }^{b}$ Number of patients undergoing each test.

$\mathrm{CO}_{2}$, carbon dioxide; UN, urea nitrogen; AST, aspartate aminotransferase; IU, international units; ALT, alanine aminotransferase; Alk, alkaline.

Liver function test abnormalities were identified in 62 women with elevated aspartate aminotransferase (AST; $15.0 \%$ of tested patients) and 53 women with elevated alanine aminotransferase (ALT; $12.9 \%$ of tested patients). However, only 11 women $(2.7 \%)$ had either an AST or an ALT $>2$ times the upper limit of normal, suggesting that the incidence of clinically relevant liver function test abnormalities is much lower. In addition, of the 95 patients with the diagnosis of ovarian cancer who underwent preoperative liver function testing, only 3 women had test results $>2$ times the upper limit of normal. With regard to preoperative abnormalities in complete blood count with platelets, 50 women $(10.6 \%)$ had elevated white blood cell counts and 54 women $(11.4 \%)$ had elevated platelets. Preoperative anemia was noted in 107 women (22.6\%).

\section{Postoperative laboratory testing abnormalities by cohort}

In the postoperative setting, 356 women had a basic metabolic panel test $(74.0 \%)$ and 27 women had a comprehensive metabolic panel test $(5.6 \%$, Table 3$)$. The incidence of laboratory result abnormalities was higher postoperatively than preoperatively. Twenty-six women $(6.8 \%)$ had elevated creatinine, and 318 women $(83.0 \%)$ had elevated glucose. In addition, $4.5 \%$ of laparoscopy patients and $5.5 \%$ of laparotomy patients had glucose above $200 \mathrm{mg} / \mathrm{dL}$ (data not shown). Electrolyte abnormalities were not infrequent in those patients tested, with hyponatremia in $8.4 \%$, hypomagnesemia in $3.6 \%$, and hypophosphatemia in $21.3 \%$. Aberrations in potassium levels were relatively uncommon on postoperative day 1 , with $4.4 \%$ and $1.6 \%$ of tested patients being hyperkalemic and hypokalemic, respectively. The laparoscopy group was significantly less likely to have hyponatremia, elevated creatinine or urea nitrogen, and hypomagnesemia on postoperative day 1 (Table 4). Despite the number of electrolyte abnormalities seen, changes were most often mild and triggered interventions such as changing intravenous fluid or electrolyte supplementation.

Almost all women $(472,98.1 \%)$ had a postoperative complete blood count with platelets tested. Hemoglobin level was below the institutional normal of $12.0 \mathrm{~g} / \mathrm{dL}$ in 355 women $(75.2 \%)$; postoperative anemia was significantly less likely in the laparoscopy group $(67.7 \%$ versus $79.1 \%$; odds ratio [OR]: $0.14 ; p=0.009)$. The more stringent criterion of hemoglobin $<7 \mathrm{~g} / \mathrm{dL}$ was then considered, given the guidelines recommending that transfusion be considered for patients whose hemoglobin fell below this threshold. ${ }^{2}$ Only 1 patient met this criterion, and she underwent a laparoscopy. This patient had a preoperative hemoglobin level of $7.3 \mathrm{~g} / \mathrm{dL}$ and a postoperative hemoglobin level of $6.2 \mathrm{~g} / \mathrm{dL}$; however, she did not receive a perioperative blood transfusion.

Elevated white blood cell counts were noted in 223 women $(47.2 \%)$. Infections were documented in 86 women $(17.9 \%)$, 


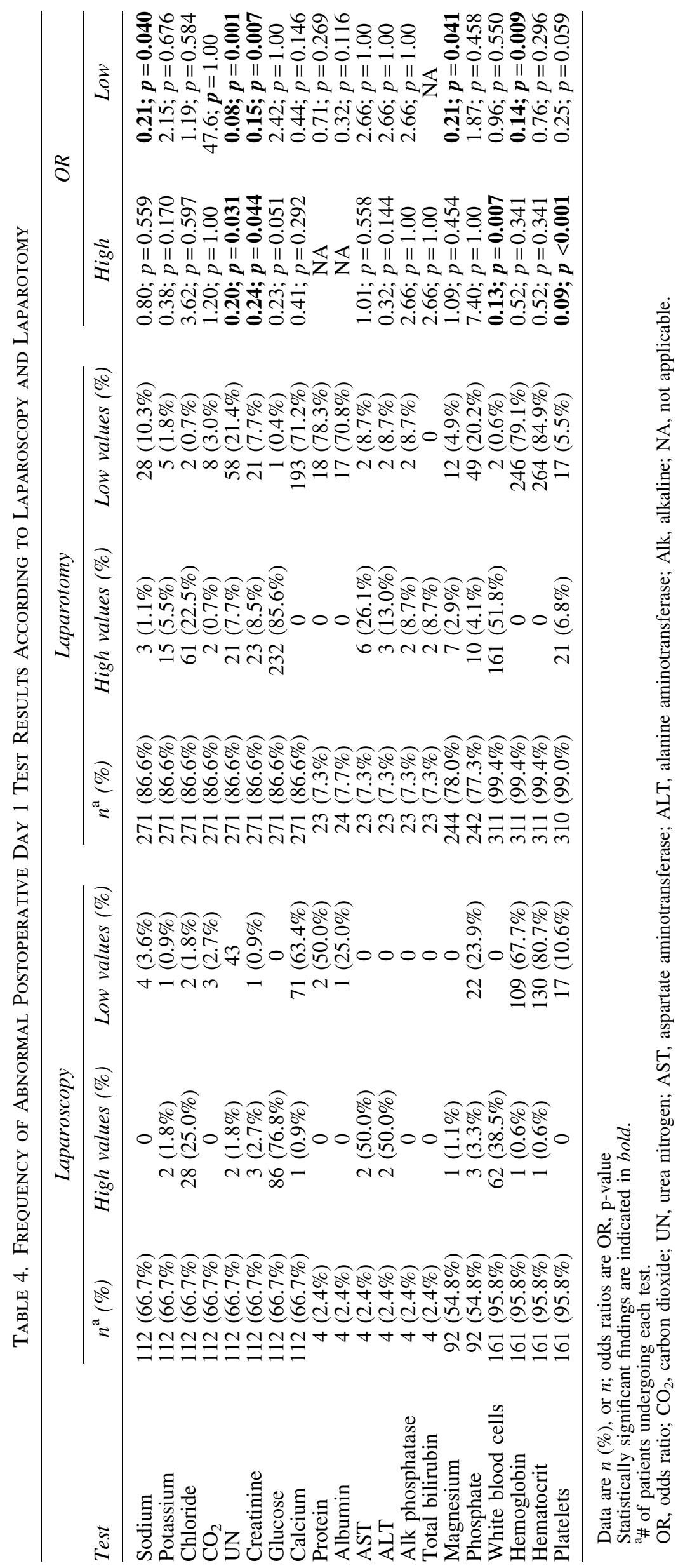


Table 5. Logistic Regression Analysis of Factors Influencing Perioperative Serum Creatinine Level

\begin{tabular}{lclcrr}
\hline Result & $\mathrm{n}^{a}$ & \multicolumn{1}{c}{ Patient factor } & OR & $95 \%$ CI & p-Value \\
\hline Preoperative Cr $>1$ & \multirow{2}{*}{458} & Diabetes & 2.42 & $1.37-4.29$ & 0.002 \\
& & ACEi/ARB medication & 3.98 & $2.38-6.65$ & $<0.001$ \\
& & Diuretic medication & 4.31 & $2.57-7.23$ & $<0.001$ \\
Postoperative $\mathrm{Cr}>1$ & \multirow{2}{*}{383} & Diabetes & 3.43 & $1.69-6.97$ & 0.001 \\
& & ACEi/ARB medication & 4.59 & $2.32-9.11$ & $<0.001$ \\
& & Diuretic medication & 4.18 & $2.12-8.26$ & $<0.001$ \\
\hline
\end{tabular}

${ }^{\mathrm{a}}$ Number of patients undergoing each test.

$\mathrm{OR}$, odds ratio; $\mathrm{CI}$, confidence interval; $\mathrm{Cr}$, serum creatinine; $\mathrm{ACEi}$, angiotensin-converting-enzyme inhibitor; ARB, angiotensin-IIreceptor blocker.

with the majority of these in the laparotomy group (laparotomy 75, laparoscopy 11; OR: 22.58; $p<0.001)$. Of these infections, $62 \%$ were of the urinary tract and $13 \%$ were of wounds. Women with elevated white blood cell counts had more than twice the odds of receiving postoperative antibiotics (OR: 2.21; 95\% confidence interval [CI]: $1.43-3.42 ; p<0.001$ ), and were at higher odds of having a documented postoperative infection (OR: $1.61 ; 95 \%$ CI: $1.01-2.59 ; p=0.047$ ) when analyzed by logistic regression.

Given the frequency of creatinine abnormalities in the perioperative period and potential implications for perioperative management, including fluid management and the dosing of renally cleared medications, the patients with creatinine abnormalities were examined further. Forty-four of $458(9.6 \%)$ women had creatinine levels $>1.0$ preoperatively; postoperatively, 26 of $390(6.8 \%)$ women had creatinine levels $>1.0$. Multiple logistic regressions were performed to determine predictors of patients with elevated serum creatinine (Table 5). Diabetes and all renally active medications were statistically significant predictors of elevated creatinine levels in both preoperative and postoperative laboratory testing.

\section{Role of laboratory abnormalities in identifying perioperative complications}

Finally, departmental quality-assurance records were examined to determine the number of patients meeting criteria for perioperative complication and if perioperative laboratory testing was a factor in identifying complications. Overall, 40/481 (8.3\%) of patients met institutional quality-assurance criteria. This included 3 laparoscopy cases (1.8\%) and 37 laparotomy cases (11.9\%; OR: 14.4; $p<0.001)$. Individual chart reviews revealed that none of the complications in these cases were initially diagnosed because of abnormalities in routine postoperative laboratory test results.

\section{Discussion}

The vast majority of patients undergoing surgery in the gynecologic oncology service at the University of Michigan had perioperative laboratory blood testing. Despite the high frequency of testing, few clinically relevant abnormal test results were obtained.

Given the above findings, the current authors propose the following guidelines for limiting perioperative laboratory testing. The current authors recommend that all patients undergo preoperative complete blood count testing for anemia and basic metabolic testing to capture both glucose and creatinine abnormalities. Postoperatively, the current authors propose no routine testing for laparoscopy patients. For laparotomy patients, given the higher rates of anemia and glucose abnormalities that were noted, the current authors recommend complete blood count testing and glucose assessment. All patients on a renally active medication should also undergo postoperative creatinine testing.

Departmental quality-assurance records were reviewed to determine if adopting the above guidelines would potentially have affected patient outcomes adversely. In reviewing the details of each specific case, it was determined that none of the cases would have had a delayed diagnosis or a worsened outcome by following the proposed recommendations.

Anemia was common preoperatively, and thus likely warrants obtaining a preoperative complete blood count with platelets to allow blood-product planning by the surgical and anesthesia teams. In patients undergoing laparotomy, the current authors were unable to identify an EBL threshold that could be used to define the subset of patients undergoing laparotomy in whom to check postoperative hemoglobin levels (data not shown). Obtaining postoperative hemoglobin levels in patients undergoing laparoscopy can be limited to those in whom there is clinical suspicion of anemia.

Electrolyte abnormalities were relatively uncommon postoperatively and often triggered interventions, such as changes to intravenous fluids and electrolyte replacement, that likely had little impact on postoperative recovery. In addition, electrolyte replacements are costly, particularly when administered intravenously. In contrast, glucose abnormalities were common, and $5.2 \%$ of patients had blood glucose values $>200 \mathrm{mg} / \mathrm{dL}$. Given the important role of glycemic control in postoperative healing, ${ }^{3}$ continued pointof-care blood glucose value monitoring is prudent in patients who are diabetic or whom undergo laparotomy and have elevated blood glucose levels postoperatively.

The implementation of new perioperative blood testing guidelines has the potential to improve many aspects of care, including decreasing perioperative laboratory fees and minimizing patient discomfort. Significant additional savings would be incurred, including eliminating unnecessary followup laboratory tests, phlebotomy fees, and electrolyte supplementation costs. In addition to gynecologic oncology patients, these findings are also applicable to the management of patients undergoing surgical intervention for benign gynecologic disease. 
While there are guidelines for preoperative testing, such as the National Institute of Clinical Excellence perioperative guidelines, ${ }^{4}$ a recent study in the benign gynecology patient population demonstrated that, in these surgical patients, the guidelines are rarely followed, with $90 \%$ of women undergoing at least one nonindicated test. ${ }^{5}$ A number of studies have examined the incidence of preoperative laboratory abnormalities in surgical patients other than the gynecology population. In one such study, the incidence of preoperative laboratory abnormalities was low and was not predictive of adverse outcomes in the postoperative period. ${ }^{6}$

Findings such as these have led to more streamlined testing for elective surgical procedures. One institution eliminated preoperative laboratory screening tests for healthy patients undergoing elective surgical procedures. ${ }^{7}$ Investigators found that patients without specific indications for testing can undergo surgery safely without preoperative laboratory tests. ${ }^{8}$ Another study examining elective surgery patients revealed that only $0.22 \%$ of the routine preoperative tests ordered revealed abnormalities that might influence perioperative management. ${ }^{9}$ These abnormalities did not result in adverse surgical or anesthetic outcomes. In addition, not only has research demonstrated that routine preoperative testing is often unnecessary based on the low incidence of abnormal test results, it also increases cost. ${ }^{10}$ One study examining the impact of new guidelines to decrease testing in the ambulatory surgery setting demonstrated that new hospital guidelines were effective for reducing preoperative testing without increasing untoward perioperative events. ${ }^{11}$

Many studies have analyzed the cost-effectiveness of preoperative type and screen testing in patients undergoing elective gynecologic surgery for benign indications, and demonstrated that such testing is not cost-effective. ${ }^{12,13}$ However, $<2.5 \%$ of the studied patients required transfusion in the perioperative period. Given the higher transfusion rate of $12 \%$ in gynecologic oncology patients undergoing laparotomy, and the possibility of conversion from laparoscopy to laparotomy intraoperatively, the current authors believe that preoperative type and screen testing should be obtained in all gynecologic oncology patients.

In the postoperative period, available guidance is more limited. Studies have demonstrated that only a small fraction of abnormal laboratory values actually trigger interventions by providers. In a study of women undergoing elective gynecologic surgery, the overall incidence of blood transfusion was $1.9 \%$, with none of these transfusions triggered specifically based on the postoperative hematocrit value. ${ }^{14}$ A more recent study of women undergoing total laparoscopic hysterectomy found routine postoperative hemoglobin testing to be of little clinical benefit in the absence of clinical signs and symptoms of anemia and that results did not guide clinical care. ${ }^{15}$

Despite the above body of research investigating preoperative and postoperative blood test frequency and incidence of abnormalities, to the current authors' knowledge no studies have been performed in patients on a gynecologic oncology service. These patients represent a unique population. From a demographic standpoint, they are women of varying ages and with a spectrum of comorbidities, both in number and type. The most similar study with regard to patient population is one that examined routine postopera- tive laboratory studies in 105 patients with colorectal cancer undergoing surgical resection. The investigators found that, although minor laboratory abnormalities were common postoperatively (12\%-67\% depending on the test), only a very small subset $(0.03 \%-5.1 \%$ depending on the test) triggered an intervention. ${ }^{16}$ Overall, those authors reported that serum potassium, hemoglobin, and serum glucose in patients with diabetes were the only values that led to therapeutic decisions. ${ }^{16}$

Limitations of the current study include its retrospective nature and the patient population represented. More than $90 \%$ of the women were Caucasian, and the average BMI was 31.8. In addition, variations in practice patterns regarding surgical aggressiveness intraoperatively for debulking and intraoperative anesthesia practices may influence the development and management of postoperative electrolyte abnormalities.

\section{Conclusions}

Future directions can include prospective cost and qualityassurance monitoring with the proposed reduction in perioperative tests to validate the safety of a reduced testing strategy. Clinically significant laboratory abnormalities are uncommon and are less likely to be found on routine perioperative testing in gynecologic oncology patients undergoing laparoscopy as compared to patients undergoing laparotomy. This suggests a role for limiting perioperative laboratory blood testing.

\section{Disclosure Statement}

No competing financial interests exist.

\section{References}

1. Mabrouk M, Frumovitz M, Greer M, et al. Trends in laparoscopic and robotic surgery among gynecologic oncologists: A survey update. Gynecol Oncol 2009;112:501.

2. Murphy MF, Wallington TB, Kelsey P, et al. Guidelines for the clinical use of red cell transfusions. Br J Haematol 2001;113:24.

3. Dronge AS, Perkal MF, Kancir S, Concato J, Aslan M, Rosenthal RA. Long-term glycemic control and postoperative infectious complications. Arch Surg 2006;141:375.

4. Reynolds TM. National Institute for Health and Clinical Excellence guidelines on preoperative tests: The use of routine preoperative tests for elective surgery. Ann Clin Biochem 2006;43:13.

5. St Clair CM, Shah M, Diver EJ, et al. Adherence to evidencebased guidelines for preoperative testing in women undergoing gynecologic surgery. Obstet Gynecol 2010;1 16:694.

6. Dzankic S, Pastor D, Gonzalez C, Leung JM. The prevalence and predictive value of abnormal preoperative laboratory tests in elderly surgical patients. Anesth Analg 2001; 93:301.

7. Narr BJ, Hansen TR, Warner MA. Preoperative laboratory screening in healthy Mayo patients: Cost-effective elimination of tests and unchanged outcomes. Mayo Clin Proc 1991;66:155.

8. Narr BJ, Warner ME, Schroeder DR, Warner MA. Outcomes of patients with no laboratory assessment before anesthesia and a surgical procedure. Mayo Clin Proc 1997; 72:505.

9. Kaplan EB, Sheiner LB, Boeckmann AJ, et al. The usefulness of preoperative laboratory screening. JAMA 1985; 253:3576. 
10. Velanovich V. Preoperative laboratory screening based on age, gender, and concomitant medical diseases. Surgery 1994;115:56.

11. Mancuso CA. Impact of new guidelines on physicians' ordering of preoperative tests. J Gen Intern Med 1999;14:166.

12. Ransom SB, McNeeley SG, Malone JM, Jr. A costeffectiveness evaluation of preoperative type-and-screen testing for vaginal hysterectomy. Am J Obstet Gynecol 1996; 175:1201.

13. Ransom SB, McNeeley SG, Hosseini RB. Costeffectiveness of routine blood type and screen testing before elective laparoscopy. Obstet Gynecol 1995;86:346.

14. Kohli N, Mallipeddi PK, Neff JM, Sze EH, Roat TW. Routine hematocrit after elective gynecologic surgery. Obstet Gynecol 2000;95:847.

15. Chamsy DJ, Louie MY, Lum DA, Phelps AL, Mansuria SM. Clinical utility of postoperative hemoglobin level test- ing following total laparoscopic hysterectomy. Am J Obstet Gynecol 2014;211:224.

16. Skenderis BS 2nd, Rodriguez-Bigas M, Weber TK, Petrelli NJ. Utility of routine postoperative laboratory studies in patients undergoing potentially curative resection for adenocarcinoma of the colon and rectum. Cancer Invest 1999;17:102.

Address correspondence to:

Karen McLean, MD, PhD

Division of Gynecologic Oncology Department of Obstetrics and Gynecology University of Michigan L4604 University South 1500 East Medical Center Drive Ann Arbor, MI 48109-5276

E-mail: karenmcl@umich.edu 